\title{
Modified stent-graft for emergent repair of blunt thoracic aortic injury
}

Piero Battocchio, MD, Michele Piazza, MD, Francesco Squizzato, MD, and Michele Antonello, PhD, Padua, Italy

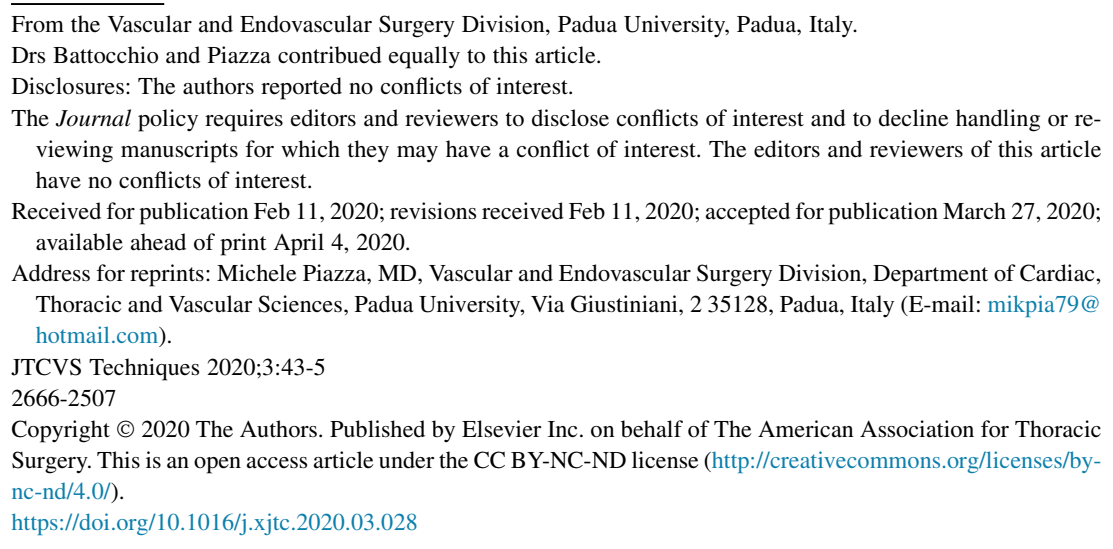

Video clip is available online.

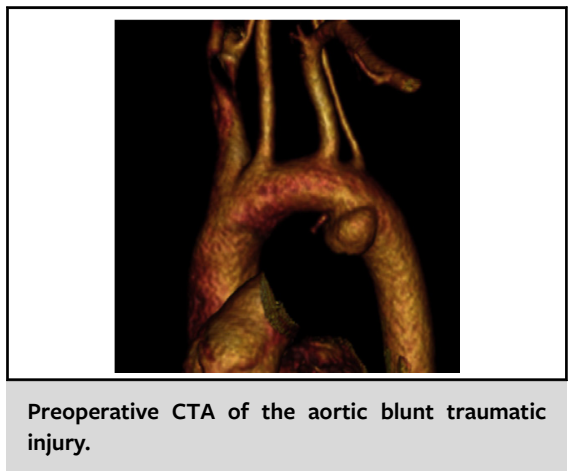

CENTRAL MESSAGE

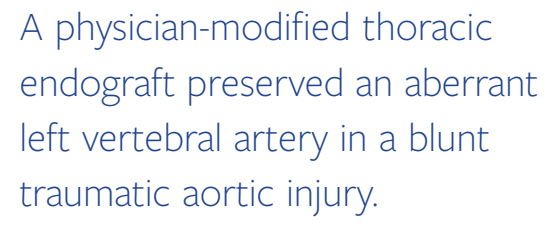

See Commentary on page 46.
A congenital anomalous origin of the left vertebral artery (LVA) directly from the aortic arch is a rare anatomical variation. ${ }^{1}$ Here, we report the case of a thoracic isthmic rupture in a young patient with this unusual anatomical condition that was successfully treated in an urgent setting with a physician-modified thoracic endograft specifically tailored to preserve LVA patency. Institutional review board approval was not required.

\section{CLINICAL SUMMARY}

A 32-year-old man was admitted after a high-energy blunt polytrauma. A computed tomography angiogram (CTA) demonstrated the presence of a grade III blunt isthmic aortic injury in the setting of independent origin of the LVA from the outer aortic arch curvature, distal to the left subclavian artery (LSA) (Figure 1, A). Because of associated cervical vertebral injuries, the patient was not eligible for any kind of open surgical debranching, and endovascular treatment was planned. The patient(s) provided informed consent for the publication of the study data.

\section{SURGICAL TECHNIQUE}

To obtain an adequate proximal seal, landing in zone 2 was mandatory, and a surgeon-modified fenestrated thoracic endograft was planned to avoid LVA coverage. A standard $25 \times 100-\mathrm{mm}$ tubular Valiant Thoracic Stent Graft (Valiant Navion; Medtronic Vascular, Santa Rosa, Calif) was selected to enable proximal and distal landing zone of at least $15 \mathrm{~mm}$; stent graft diameter was oversized by $10 \%$ to $15 \%$. A $10 \times 25-\mathrm{mm}$ fenestration was created; to calculate the length of the fenestration, we measured the distance between the proximal origin of the LSA and the distal end of the LVA $+3 \mathrm{~mm}$ on all sides; to calculate the width, we measured the larger diameter between the LSA and the LVA (it was that of the LSA) $+1 \mathrm{~mm}$ on all sides (Figure 2, $A$ and $B$ ).

The device was prepared on a side table after partial unsheathing (Figure 2, C). A no. 11 blade scalpel was used to fashion the fenestration, which was then reinforced circumferentially with the loop of a snare (Amplatz Goose Neck Snare; Medtronic Vascular) secured on the fabric with a multiple 5-0 polypropylene single locked suture. After resheathing, the endograft was oriented outside the patient and then deployed at the desired position in the aortic arch (Video 1).

The postoperative CTA (Figure 1, B) demonstrated the exclusion of the rupture, with residual leak between the graft fabric and the lateral aortic wall related to incomplete apposition of the fenestration skeleton. A secondary 


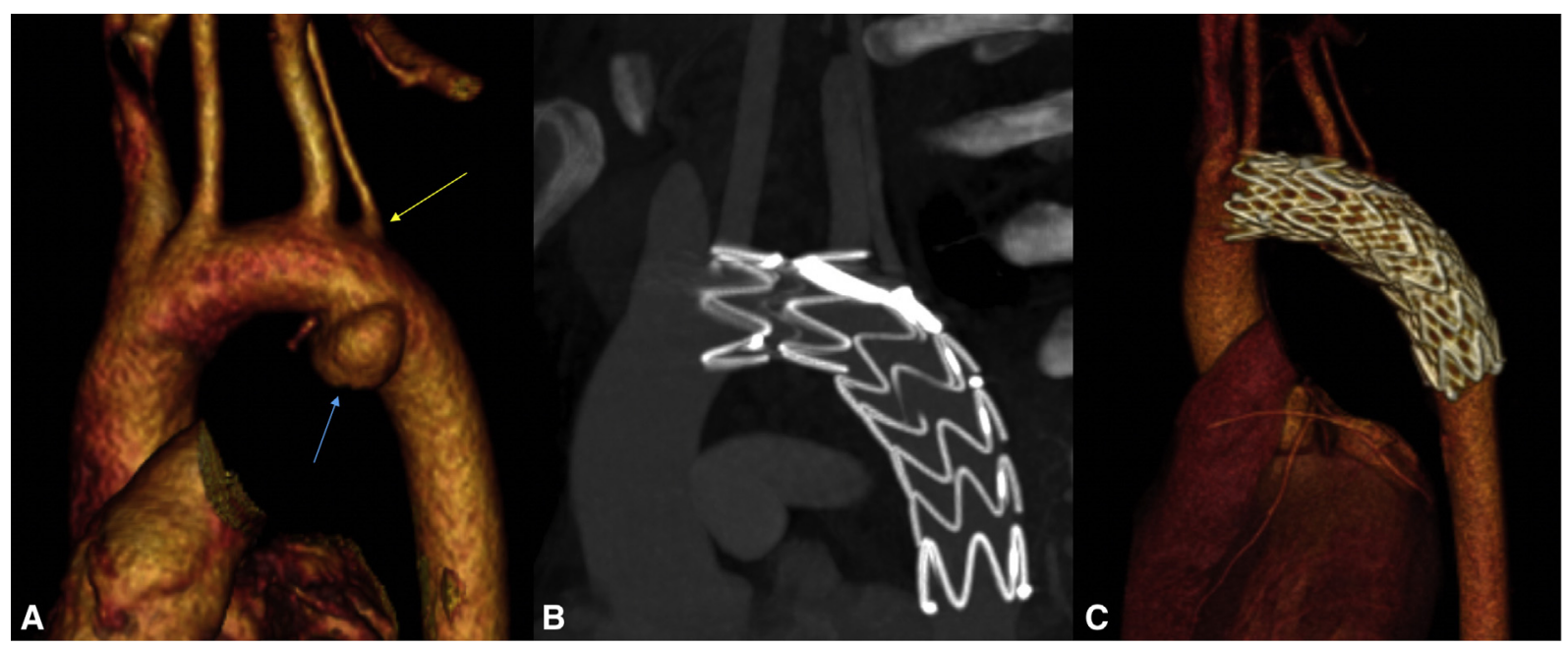

FIGURE 1. A, 3D reconstruction of the preoperative CT angiography showing the grade III aortic blunt injury at the level of the small curvature of the aortic arch (blue arrow). The anomalous left vertebral artery arising from the aortic arch is indicated by the yellow arrow. B, Multiplanar reconstruction of the postoperative angio-CT scan, showing good positioning of the fenestration of the physician-modified graft, with preserved patency to both the LSA and LVA. C, 3D reconstruction of the final angio-CT, after deployment of the aortic nitinol bare-metal stent. Note the "open fenestration," including the origin of the LSA and LVA, with preserved patency of the aortic branches.

procedure was then performed; a $28 \times 24$-mm bare-metal nitinol aortic stent (E-XL; Jotec GmbH, Hechingen, Germany) was deployed over the previous endograft, followed by stent-graft ballooning, to obtain an adequate apposition of the graft fabric to the aortic wall and stabilize the open fenestration. The CTA performed after this second procedure (Figure 1, C) demonstrated the regular patency of all the supra-aortic vessels and the complete exclusion of the rupture with no signs of residual endoleak. CTA at 6 months postoperatively demonstrated the absence of any graft-related complication. The patient provided informed consent for the publication of this study data.

\section{DISCUSSION}

Even if intentional LSA coverage without revascularization is feasible in emergency setting, ${ }^{2}$ spinal cord and left upper limb ischemia, as well as vertebral artery territory stroke, are possible associated complications both in the early $^{2}$ and mid-term period. ${ }^{1}$ An anatomic variation of the LVA originating directly from the arch may increase the

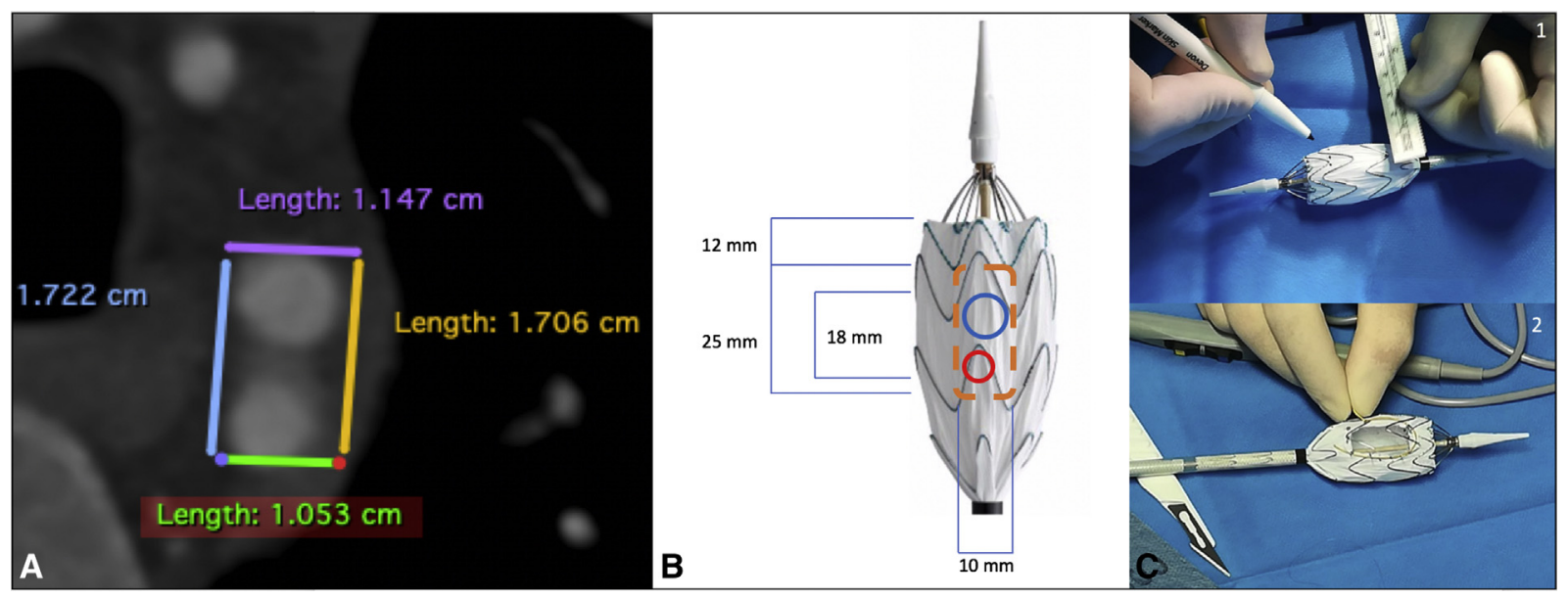

FIGURE 2. A, Axial cut of the CT angiography scan showing the preoperative planning of the open fenestration, to include the origin of both the LVA and LSA. B, Sizing of the surgeon-modified graft. To calculate the length of the "open fenestration," $3 \mathrm{~mm}$ was added on each side to the distance between the proximal origin of the LSA and the distal end of the LVA. To calculate the width of the "open fenestration," 1 mm was added on each side compared with the LSA diameter. C, Preparation of the surgeon-modified graft. After partial deployment, the "open fenestration" is outlined on the stent graft fabric using a ruler and sterile marking pen. The fenestration is reinforced with the loop of a snare, then the modified stent-graft is reloaded in the existing sheath using a temporary looped strand to collapse each stent. 


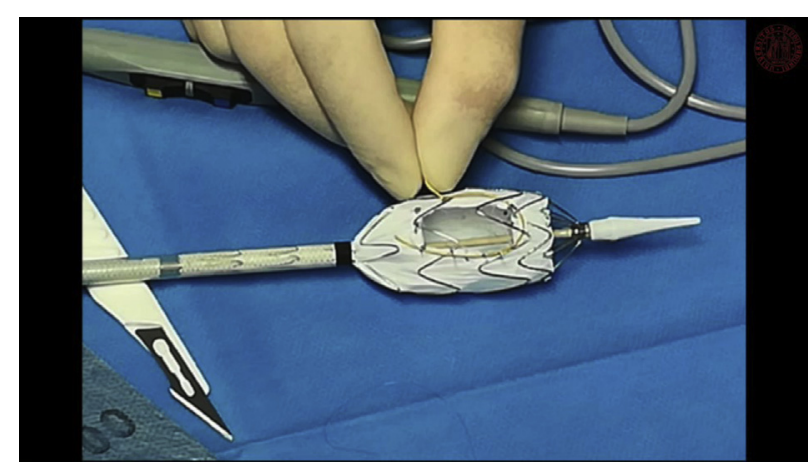

VIDEO 1. Video summary of the case showing the preoperative computed tomography, planning, device preparation and implantation, and the final result. Video available at: https://www.jtcvs.org/article/S2666-2507(20) 30180-2/fulltext.

risk of vertebral stroke during thoracic endovascular aortic repair. In an emergency setting, other total endovascular approaches preserving LSA patency include the chimney or periscope technique ${ }^{3}$ or retrograde laser fenestration. ${ }^{4}$ However, these options were not adaptable in this case because of the small diameter of the LVA, the short distance between origin of the LSA and LVA, and an unfavorable anatomy for a retrograde access. The use of a custom-made fenestrated arch device was not indicated in this case because of the emergent setting.

Homemade proximal fenestrated stent-graft for thoracic endovascular aortic repair of zone 2 acute aortic syndrome has already been reported, ${ }^{5}$ showing its feasibility and efficacy. The choice of a Medtronic device was based on this previous experience and on the immediate availability of the desired size in our internal endovascular depot. In case of blunt traumatic aortic injuries, an oversizing ranging from $10 \%$ to $20 \%$ is usually used in our center, depending on the hemodynamic status of the patient; in this specific case, the patient was hemodynamically stable and a $10 \%$ to $15 \%$ oversizing was considered appropriate; the decision to use a physician-modified graft did not affect this choice. Our technique seems to slightly differ from previous reports, because this case was planned with the large ("open") fenestration located only $13 \mathrm{~mm}$ from the proximal edge of the stent-graft, and with the aim to incorporate both the LSA and LVA, without the use of any bridging stent.

The use of the E-XL nitinol stent as distal uncovered self-expanding nitinol stent in the PETTICOAT technique has already been reported. Other alternative options include the Zenith Dissection Endovascular stent (Cook Medical Inc, Bloomington, Ind) and the Sinus-XL Stent (optimed Medical Instruments $\mathrm{GmbH}$, Ettlingen, Germany). Its advantages are that it adapts to different aortic diameters and its radial force promotes successful positive remodeling; in this case, it was specifically chosen to promote a complete and durable apposition of the previously implanted thoracic endograft to the aortic arch, maintaining supra-aortic vessels patency.

\section{CONCLUSIONS}

This case demonstrates that in selected emergent cases of focal thoracic aortic injury, a surgeon-modified endograft with an "open fenestration" supported by a bare metal nitinol stent is a feasible option to exclude the rupture and preserve the patency of supra-aortic vessels arising from the outer curvature of the arch.

\section{References}

1. Piazza M, Squizzato F, Milan L, Miccoli T, Grego F, Antonello M; Global Registry for Endovascular Aortic Treatment (GREAT) investigators. Incidence and predictors of neurological complications following thoracic endovascular aneurysm repair in the global registry for endovascular aortic treatment. Eur J Vasc Endovasc Surg. 2019;58:512-9.

2. Riambau V, Böckler D, Brunkwall J, Cao P, Chiesa R, Coppi G, et al. Editor's Choice - management of descending thoracic aorta diseases. Eur J Vasc Endovasc Surg. 2017;53:4-52.

3. Baldwin ZK, Chuter TA, Hiramoto JS, Reilly LM, Schneider DB. Double-barrel technique for preservation of aortic arch branches during thoracic endovascular aortic repair. Ann Vasc Surg. 2008;22:703-9.

4. Redlinger RE, Ahanchi SS, Panneton JM. In situ laser fenestration during emergent thoracic endovascular aortic repair is an effective method for left subclavian artery revascularization. J Vasc Surg. 2013;58:1171-7.

5. Canaud L, Baba T, Gandet T, Narayama K, Ozdemir BA, Shibata T, et al Physician-modified thoracic stent-grafts for the treatment of aortic arch lesions. J Endovasc Ther. 2017;24:542-8. 